\title{
Assessment of the Immune Effect and Safety of Cell Culture-Derived Influenza Vaccine from Egg-Derived by Meta Analysis
}

\author{
Xingliang Geng, Ze Liu, Guoyang Liao * \\ Institute of Medical Biology, Chinese Academy of Medical Science and Peking Union Medical College; Kunming, Yunnan \\ Province, China; Zip Code: 650118.
}

Corresponding author: Guoyang Liao; mybelieze@126.com

Received 02 September 2020;

Accepted 25 September 2020;

Published 04 October 2020

\begin{abstract}
Objective: The study was to evaluate the immune effect and safety difference of cell culture-derived influenza vaccine from egg-derived by meta analysis; Methods: It selected the article about clinical trials through medline, embase, cochrane library, FEBM, clinicaltrials.gov, WHO, and International Standard Randomised Controlled Trial Number Registry. There was no language restrictions (update to Apr 25, 2016). The

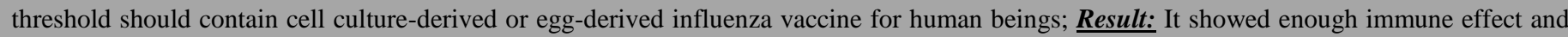
accepted safety by using cell culture-derived influenza vaccine, compared with egg-derived influenza vaccine; $\underline{\text { Conclusion: }}$ The cell culturederived influenza vaccine could be used for human after clinical trials, not differ from egg-derived.
\end{abstract}

Keywords: meta; influenza vaccine; cell culture; egg;

\section{Dear editor,}

Here, we reported the assessment of cell culture-derived influenza vaccine (CCIV) from egg-derived by meta analysis, according to immune effect and safety. Since vaccination against influenza began in the 1930s with large scale availability in the United States beginning in 1945, it was almost 75-year using egg-derived influenza vaccine. Although it was a classical and effective method, the conventional egg-based manufacturing technology for seasonal influenza vaccines has several drawbacks, including inflexibility, viral mutations, inadequate supply egg, risk of contamination, and so on ${ }^{[1,2]}$. In 1995, the World Health Organization (WHO) recommended to develop alternative influenza virus cultivation systems, specifically, to explore promising mammalian cell culture lines(Cell culture as a substrate for the production of influenza vaccines: Memorandum from a WHO meeting in 1995).

Cell-culture-derived influenza vaccine production methods could provide benefits over classical embryonated-egg technology, including a higher production capacity and the enough supply ${ }^{[3,4]}$. This study was to integrate the available evidence and to find identify whether there was difference between the two methods derived, about its safety and immunogenicity.

It selected the articles about clinical trials through medline, embase, cochrane library, FEBM, clinicaltrials.gov, WHO, and International Standard Randomised Controlled Trial Number
Registry. There was no language restrictions (update to Apr 25, 2016). The threshold themes should contain cell culture-derived or egg-derived influenza vaccine for human beings. There were 89 articles included in this meta analysis. 18 of records identified through Embase databse searching; 31 of additional records identified through Pubmed; 10 of records identified through the Cochrane central register of controlled trials; 15 of additional records identified through clinical trials.gov; 15 of records identified through FEBM database searching. After removing the duplication, 34 of them were left. According to the relativities of the abstract, only 18 essay were used to search for full text.

The references of all relevant articles, including reviews, were checked for further analysis. Importantly, we focused on randomized trials. If the article studied with single vaccine formulation, or tested individuals non-randomly, it wasn't included in quantitative syntheses. Finally, we decided to perform the metaanalysis on 5 studies by screened the 18 full text ${ }^{[1,5-8]}$.

The meta-analysis was done using RevMan (version 5.3) analyzing software. All articles heterogeneity was assessed using a chi-square-based Q statistic. The $\mathrm{p}$-value of $\leq 0.05$ was considered statistically significant. If there was significant heterogeneity, outcome data from the trials were analyzed using a random effects model (DerSimonian-Laird) ${ }^{[3,4,9]}$ to estimate the pooled RR. In the absence of heterogeneity, meta-analysis was performed using a fixed effects model (Mantel-Haenszel). Calculating the merger 
ratio (odds ratio, OR) and $95 \%$ confidence area (confidence interval, 95\% CI) were evaluated.

As for immunogenicity assessment, the seroconversion was the core concern. According to the Guidance for Industry: Clinical Data Needed to Support the Licensure of Seasonal Inactivated Influenza Vaccines, appropriate endpoints might be the hemagglutination inhibition (HI) antibody responses to each viral strain included in vaccine.

Rates of seroconversion, defined as the percentage of subjects with either a pre-vaccination $\mathrm{HI}$ titer $<1: 10$ and a postvaccination $H I$ titer $\geq 1: 140$ or a pre-vaccination $H I$ titer $\geq 1: 10$ and a minimum four-fold rise in post -vaccination $\mathrm{HI}$ antibody titer.

In those researches, if both seroconversion and seroresponse data were available, only seroconversion data were used, because they might be cut-off the baseline status (seroresponse also includes individuals with pre-vaccination immunity).

It showed the immunogenicity of cell culture-derived compared with egg-derived influenza vaccines in Figure I. The HI antibody seroconversion rate was calculated between these two groups. It found that there was no significant difference between these two groups, respectively, for H1N1, H3N2 and B Sub-types.

Since many studies reported mild and moderate adverse events together, they were divided into two different groups. On the other hand, many studies reported adverse events after the first and second dose separately, but reported together in some articles. In this analysis, we selected the higher data during the papers. In the end, the funnel plot to evaluate publication bias (Figure II).
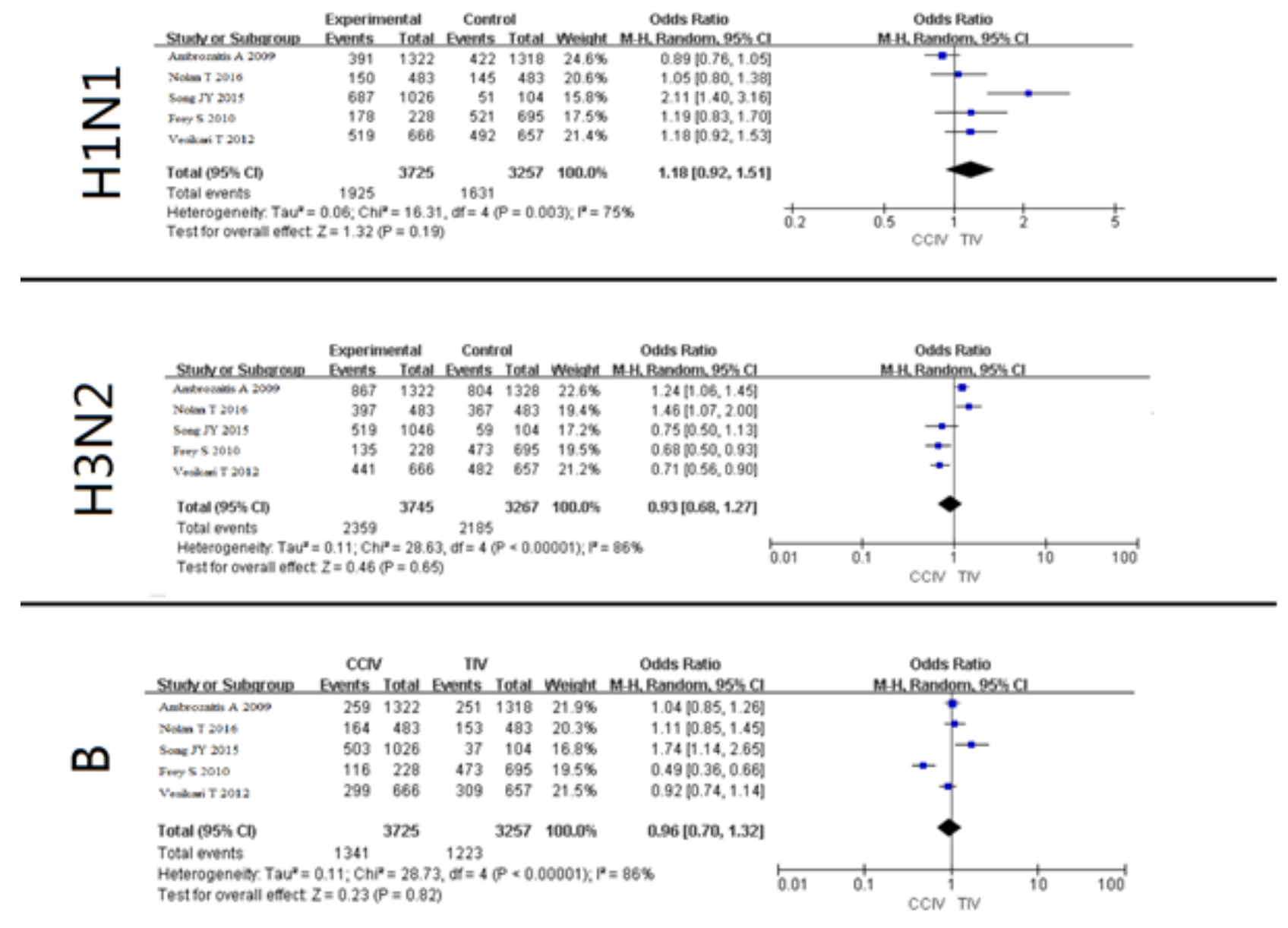

Figure1: The immunogenicity of cell culture-derived compared with egg-derived influenza vaccines 

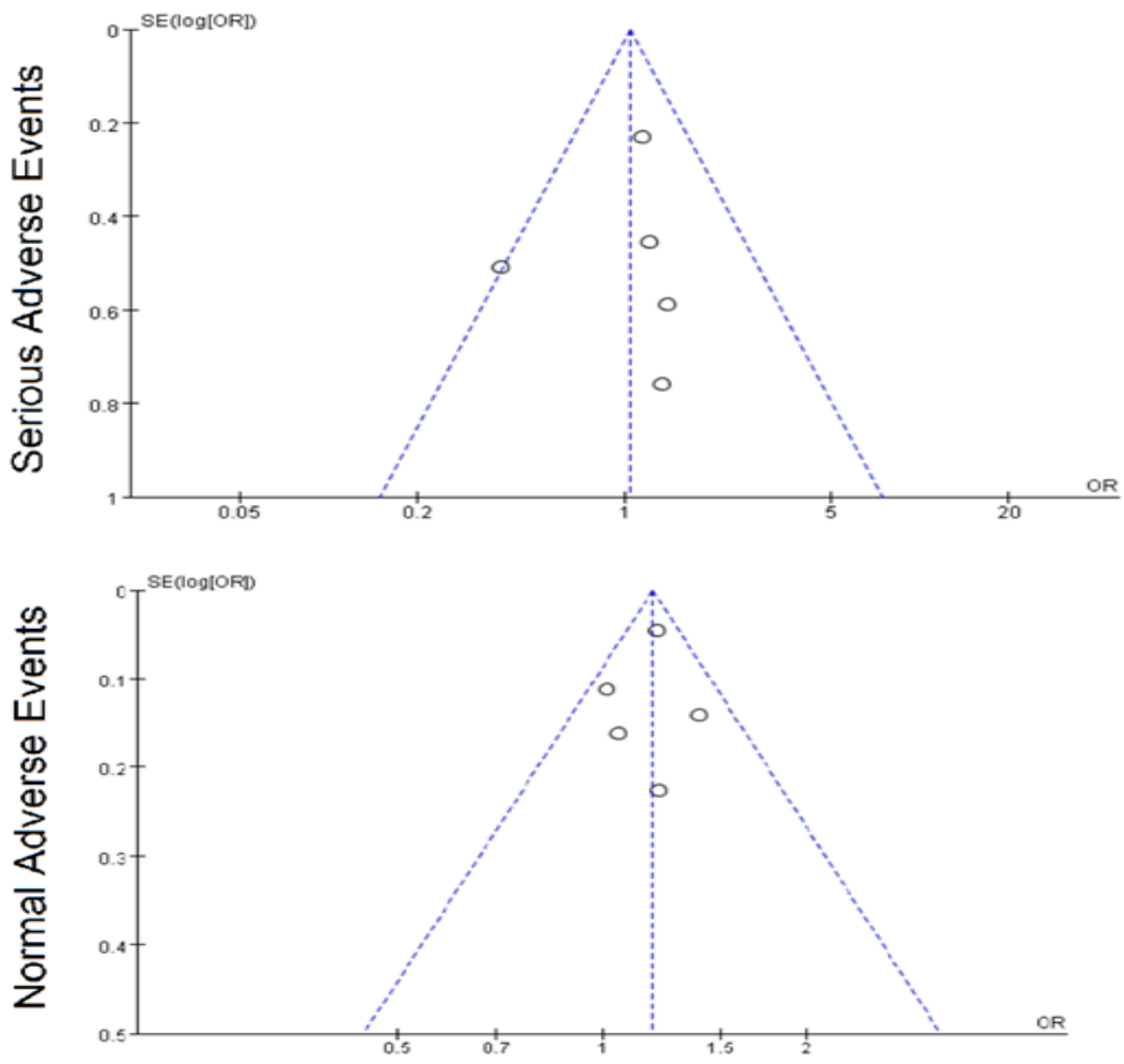

Figure2: The funnel plot to evaluate publication bias

As for adverse events about safety, there were some clinical symptoms, including fever, headache, myalgia or muscle aches, malaise or fatigue (systemic reactions), injection-site pain and erythema or redness (local reactions). Those were defined as normal adverse events. Simultaneously, the serious adverse events were including shock, syncope, epilepsy, respiratory arrest and heart failure. After analysis, there was no significant difference in both normal and serious adverse events between two groups.

In summary, although the egg-derived influenza vaccine had been used for several decades, the cell culture-derived is a trend for future development of influenza vaccine. Due to the insufficient supply of embryonated chicken eggs, the preparation of large quantities of inactivated influenza vaccines will require an alternative virus culture system after the emergence or reemergence of a pandemic influenza virus ${ }^{[2,10-12]}$. In this meta-analysis, it compared cell culture-derived with egg-derived influenza vaccine, in clinical trails but not experimental details. There were 5 valuable articles update to update to Apr 25, 2016, about that theme. After Systematic Analysis, it found that the cell culture-derived influenza vaccine could stimulate the body to produce antibodies, which was enough for protection efficiency. Their seroconversion was consistent with egg-derived influenza vaccine results. Additionally, those results were steady for each three subtypes. The HI antibody titers weren't low compared with the egg-derived group. As for safety assessment, there was no significant difference in both normal and serious adverse events between two groups. However, in the egg-derived group, it induced shock for one case. So it might be made a conclusion that cell culture-derived influenza vaccine had enough immunogenicity and evidenced safety. The new trend influenza vaccine should be strongly promoted and applied for population.

\section{Conclusion}

In this study, the immunogenicity and safe of culture-based and egg-raised inactivated viral influenza strains (H1N1, H3N2, B) was assessed by meta-analysis (Chi square/M-H), which provided additional support for the potential clinical application of the cell culture-based vaccine.

\section{Funding}

This work was supported by CAMS Innovation Fund for Medical Sciences (CIFMS) (2016-I2M-3-026); Innovation team in Yunnan Province (2015HC027); Yunnan Applied Basic Research Project (2017FB039).

\section{References}

[1] Nolan T, Chotpitayasunondh T, Capeding MR, Carson S, Senders SD, Jaehnig P, de Rooij R7, Chandra R.Safety and tolerability of a cell culture derived trivalent subunit inactivated influenza vaccine administered tohealthy children and adolescents: A Phase III, randomized, multicenter, observer-blind study.2016.Vaccine,34(2):230-6.

[2] Schuind A, Segall N, Drame M, Innis BL.Immunogenicity and Safety of an EB66 Cell-CultureDerived Influenza A/Indonesia/5/2005(H5N1) AS03- 
Adjuvanted Vaccine: A Phase 1 Randomized Trial. 2015.J Infect Dis,212(4):531-41.

[3] Girard MP, Katz J, Pervikov Y, Palkonyay L, Kieny MP.Report of the 6th meeting on the evaluation of pandemic influenza vaccines in clinical trials World HealthOrganization, Geneva, Switzerland, 17-18 February 2010. 2010. Vaccine,28(42):6811-20.

[4] Nakowitsch S, Waltenberger AM, Wressnigg N, Ferstl N, Triendl A, Kiefmann B, Montomoli E, Lapini G, Sergeeva M, Muster T, Romanova JR.Egg- or cell culture-derived hemagglutinin mutations impair virus stability and antigen content of inactivatedinfluenza vaccines.2014.Biotechnol J,9(3):405-14.

[5] Ambrozaitis A, Groth N, Bugarini R, Sparacio V, Podda A, Lattanzi M.A novel mammalian cell-culture technique for consistent production of a well-tolerated and immunogenictrivalent subunit influenza vaccine.2009.Vaccine, 27(43):6022-9.

[6] Song JY, Cheong HJ, Lee J, Woo HJ, Wie SH, Lee JS, Kim SW, Noh JY, Choi WS, Kim H, Kim KH, Kim WJ.Immunogenicity and safety of a cell culture-derived inactivated trivalent influenza vaccine (NBP607): A randomized, double-blind, multi-center, phase 3 clinical trial.2015.Vaccine,33(41):5437-44.

[7] Frey S, Vesikari T, Szymczakiewicz-Multanowska A, Lattanzi M, Izu A, Groth N, Holmes S.Clinical efficacy of cell culture-derived and egg-derived inactivated subunit influenza vaccines in healthyadults.2010.Clin Infect Dis,51(9):997-1004.
[8] Vesikari T, Block SL, Guerra F, Lattanzi M, Holmes S, Izu A, Gaitatzis N, Hilbert AK, Groth N.Immunogenicity, safety and reactogenicity of a mammalian cell-culture-derived influenza vaccine in healthy children and adolescents three to seventeen years of age.2012.Pediatr Infect Dis J,31(5):494-500.

[9] Barrett PN, Berezuk G, Fritsch S, Aichinger G, Hart MK, El-Amin W, Kistner O, Ehrlich HJ.Efficacy, safety, and immunogenicity of a Vero-cell-culture-derived trivalent influenza vaccine: a multicentre, doubleblind,randomised, placebo-controlled trial. 2011.Lancet,377(9767):751-9.

[10] Groth N, Montomoli E, Gentile C, Manini I, Bugarini R, Podda A.Safety, tolerability and immunogenicity of a mammalian cell-culture-derived influenza vaccine: a sequentialPhase I and Phase II clinical trial.2009.Vaccine,27(5):786-91.

[11] Manini I, Domnich A, Amicizia D, Rossi S, Pozzi T, Gasparini R, Panatto D, Montomoli E.Flucelvax (Optaflu) for seasonal influenza.2015.Expert Rev Vaccines, 14(6):789-804.

[12] Szymczakiewicz-Multanowska A, Lattanzi M, Izu A, Casula D, Sparacio M, Kovacs C, Groth N.Safety assessment and immunogenicity of a cell-culture-derived influenza vaccine in adults and elderly subjects over three successive influenza seasons.2012.Hum Vaccin Immunother,8(5):645-52. 